\title{
A NOTE ON SOME INEQUALITIES OF MARTINGALE SHARP FUNCTIONS
}

\author{
REN YANBO
}

Abstract. In this paper, some new inequalities for the sharp functions of martingales are established by use of rearrangement technique.

Mathematics subject classification (2010): 60G42, 46E45.

Keywords and phrases: Martingale inequality, rearrangement function, sharp function.

\section{REFERENCES}

[1] F. WeIsZ, Martingale Hardy Spaces and their Applications in Fourier Analysis, Lecture Notes in Math, Vol. 1568, Spring-Verlag, 1994.

[2] R. L. LoNG, Rearrangement techniques in martingale setting, Illinois J. Math. 35 (1991), 506-521.

[3] R. L. Long, Martingale spaces and inequalities, Peking Univ Press, Beijing, 1993.

[4] R. J. Bagby, D. S. KurTZ, A rearranged good $\lambda$-inequality, Tran. Amer. Math. Soc. 293 (1986), $71-81$.

[5] J. BERGH, J. LÖFSTRÖM, Interpolation Spaces, Springer-Verlag, Berlin, 1976. 